\title{
Introduction to the Fourth Annual Lifelog Search Challenge, LSC'21
}

\author{
Gurrin, Cathal ; Jónsson, Björn bóR ; Schöffmann, Klaus ; Dang-Nguyen, Duc-Tien ; Lokoč, Jakub ;
} Tran, Minh-Triet ; Hürst, Wolfgang ; Rossetto, Luca ; Healy, Graham

\begin{abstract}
The Lifelog Search Challenge (LSC) is an annual benchmarking challenge for comparing approaches to interactive retrieval from multi-modal lifelogs. LSC'21, the fourth challenge, attracted sixteen participants, each of which had developed interactive retrieval systems for large multimodal lifelogs. These interactive retrieval systems participated in a comparative evaluation in front of an online live-audience at the LSC workshop at ACM ICMR'21. This overview presents the motivation for LSC'21, the lifelog dataset used in the competition, and the participating systems.
\end{abstract}

DOI: https://doi.org/10.1145/3460426.3470945

Posted at the Zurich Open Repository and Archive, University of Zurich ZORA URL: https://doi.org/10.5167/uzh-206331

Conference or Workshop Item

Published Version

Originally published at:

Gurrin, Cathal; Jónsson, Björn póR; Schöffmann, Klaus; Dang-Nguyen, Duc-Tien; Lokoč, Jakub; Tran, Minh-Triet; Hürst, Wolfgang; Rossetto, Luca; Healy, Graham (2021). Introduction to the Fourth Annual Lifelog Search Challenge, LSC'21. In: ICMR '21: International Conference on Multimedia Retrieval, Taipei Taiwan, 21 September 2021 - 24 September 2021. ACM, 690-691.

DOI: https://doi.org/10.1145/3460426.3470945 


\section{Introduction to the Fourth Annual Lifelog Search Challenge, LSC'21}

\author{
Cathal Gurrin \\ Dublin City University \\ Ireland \\ Duc-Tien Dang-Nguyen \\ University of Bergen \\ Kristiania University College \\ Norway \\ Wolfgang Hürst
Utrecht University
Netherlands
}

\begin{abstract}
The Lifelog Search Challenge (LSC) is an annual benchmarking challenge for comparing approaches to interactive retrieval from multi-modal lifelogs. LSC'21, the fourth challenge, attracted sixteen participants, each of which had developed interactive retrieval systems for large multimodal lifelogs. These interactive retrieval systems participated in a comparative evaluation in front of an online live-audience at the LSC workshop at ACM ICMR'21. This overview presents the motivation for LSC'21, the lifelog dataset used in the competition, and the participating systems.
\end{abstract}

\section{CCS CONCEPTS}

- Human-centered computing $\rightarrow$ Empirical studies in interaction design; • Information systems $\rightarrow$ Mobile information processing systems; Search interfaces.

\section{KEYWORDS}

Lifelog, Interactive Retrieval Systems, Benchmarking

\section{ACM Reference Format:}

Cathal Gurrin, Björn Pór Jónsson, Klaus Schöffmann, Duc-Tien Dang-Nguyen, Jakub Lokoč, Minh-Triet Tran, Wolfgang Hürst, Luca Rossetto, and Graham Healy. 2021. Introduction to the Fourth Annual Lifelog Search Challenge, LSC'21. In Proceedings of the 2021 International Conference on Multimedia Retrieval (ICMR '21), August 21-24, 2021, Taipei, Taiwan. ACM, New York, NY, USA, 2 pages. https://doi.org/10.1145/3460426.3470945

\section{INTRODUCTION}

Since the MyLifeBits project [6], there has been an increasing level of research attention to the topic of lifelog data analysis, management and search. This interest initiated the Lifelog Search Challenge (LSC) comparative benchmarking workshop that began in

Permission to make digital or hard copies of part or all of this work for personal or classroom use is granted without fee provided that copies are not made or distributed for profit or commercial advantage and that copies bear this notice and the full citation on the first page. Copyrights for third-party components of this work must be honored

For all other uses, contact the owner/author(s).

ICMR '21, August 21-24, 2021, Taipei, Taiwan

(C) 2021 Copyright held by the owner/author(s)

ACM ISBN 978-1-4503-8463-6/21/08.

https://doi.org/10.1145/3460426.3470945

\author{
Klaus Schöffmann \\ Klagenfurt University \\ Austria \\ Minh-Triet Tran \\ VNU HCM - University of Science \\ Vietnam
}

\author{
Graham Healy \\ Dublin City University \\ Ireland
}


scores of the topics up until that point. For more details of scoring mechanisms, see the review of LSC'18 [8].

\section{PARTICIPATING SYSTEMS}

Sixteen teams took part in LSC'21, meaning that sixteen interactive lifelog retrieval engines joined the challenge and live competition.

Many of the teams presented enhanced versions of systems from previous LSC workshops. The MyScéal retrieval system, developed to explore query expansion and word embedding approaches to interactive retrieval [20], participated with an enhanced version of the top-performing system from LSC'20. The SOMHunter system [13], successful at LSC'20 with temporal queries, relevance score updates with feedback and SOM-based screens, included more visual data search capabilities and an effective text-image retrieval model recently released by OpenAI. The Exquisitor scalable media exploration system [10] was enhanced for LSC'21 to better support tasks with a temporal component by adding features that allowed the user to build multiple classifiers and merge their results. Likewise the LifeGraph system [15] presented a new-iteration of the knowledge-graph-based retrieval engine incorporating enhanced retrieval facilities and a revised underlying graph traversal mechanism. The vitrivr system [9] introduced enhancements, such as image stabilisation to reduce image degradation caused by lifelogger movements for improved concept detection. The lifeXplore system [12] featured improvements to handle temporal queries and provide advanced day summary features. LifeSeeker 3.0 [14] featured enhancements to visual and location metadata, visual similarity matching and improved text ranking. Similarly, Voxento 2.0 [2] was improved by the addition of dynamic result filtering and a better retrieval engine. A technique based on concept recommendation [3] was also introduced based on multiple visual concept enhancements to an existing LSC'20 system. Finally, FIRST 2.0 [21] included a totally redesigned flexible query system.

There were a number of new systems developed for LSC'21. One such system was vitrivr-VR [18] which, based on the vitrivr system, leveraged VR to offer novel retrieval and user interaction models. The ViRMA virtual reality lifelog retrieval tool [4], was another VR system, which introduced an effective representation of large volume multimedia data in the VR space. Another new system, Memento [1], leveraged semantic representations of images and textual queries projected into a common latent space, while PhotoCube [17] mapped lifelog data into a hypercube in a multidimensional metadata space, which was then filtered and projected onto a 3D space. Finally LifeMon [5] explored the efficient use of MongoDB document stores for indexing and XQC [11] presented a novel interactive learning interface running on mobile devices.

\section{ACKNOWLEDGMENTS}

This publication has emanated from research supported in part by Science Foundation Ireland under grant numbers SFI/12/RC/2289$\mathrm{P} 2$ and SFI/13/RC/2106-P2, the Czech Science Foundation (GAČR) project 19-22071Y, and co-funded by the European Regional Development Fund.

\section{REFERENCES}

[1] Naushad Alam, Yvette Graham, and Cathal Gurrin. 2021. Memento: A Prototype Lifelog Search Engine for LSC'21.. In Proceedings of the 2021 ACM Workshop on the Lifelog Search Challenge, LSC21. Taipei, Thailand.

[2] Ahmed Alateeq, Mark Roantree, and Cathal Gurrin. 2021. Voxento 2.0: A Prototype Voice-controlled Interactive Search Engine for Lifelog. In Proceedings of the 2021 ACM Workshop on the Lifelog Search Challenge, LSC21. Taipei, Thailand.

[3] Wei-Hong Ang, An-Zi Yen, Tai-Te Chu, Hen-Hsen Huang, and Hsin-Hsi Chen. 2021. An Interactive Approach for Multimodal Lifelog Retrieval through Concept Recommendation. In Proceedings of the 2021 ACM Workshop on the Lifelog Search Challenge, LSC21. Taipei, Thailand.

[4] Aaron Duane and Björn Pór Jónsson. 2021. ViRMA: Virtual Reality Multimedia Analytics at LSC 2021. In Proceedings of the 2021 ACM Workshop on the Lifelog Search Challenge, LSC21. Taipei, Thailand.

[5] Alexander Christian Faisst and Björn Pór Jónsson. 2021. LifeMon: A MongoDBBased Lifelog Retrieval Prototype. In Proceedings of the 2021 ACM Workshop on the Lifelog Search Challenge, LSC21. Taipei, Thailand.

[6] Jim Gemmell, Gordon Bell, and Roger Lueder. 2002. MyLifeBits: fulfilling the Memex vision. Proceedings of the tenth ... FIX IT (2002), 235-238.

[7] Cathal Gurrin, Tu-Khiem Le, Van-Tu Ninh, Duc-Tien Dang-Nguyen, Björn Pór Jónsson, Jakub Lokoč, Wolfgang Hürst, Minh-Triet Tran, and Klaus Schöffmann. 2020. Introduction to the Third Annual Lifelog Search Challenge (LSC'20). In Proceedings of the 2020 International Conference on Multimedia Retrieval (Dublin, Ireland) (ICMR '20). Association for Computing Machinery, New York, NY, USA, $584-585$.

[8] Cathal Gurrin, Klaus Schoeffmann, Hideo Joho, Andreas Leibetseder, Liting Zhou, Aaron Duane, Dang Nguyen, Duc Tien, Michael Riegler, Luca Piras, et al. 2019. Comparing approaches to interactive lifelog search at the lifelog search challenge (LSC2018). ITE Transactions on Media Technology and Applications 7, 2 (2019), 46-59.

[9] Silvan Heller, Ralph Gasser, Sanja Popovic, Luca Rossetto, Loris Sauter, Florian Spiess, Heiko Schuldt, and Mahnaz Parian. 2021. Interactive Multimodal Lifelog Retrieval with vitrivr at LSC 2021. In Proceedings of the 2021 ACM Workshop on the Lifelog Search Challenge, LSC21. Taipei, Thailand.

[10] Omar Shahbaz Khan, Björn Pór Jónsson, Aaron Duane, Jan Zahálka, Stevan Rudinac, and Marcel Worring. 2021. Exquisitor at the Lifelog Search Challenge 2021: Relationships Between Semantic Classifiers. In Proceedings of the 2021 ACM Workshop on the Lifelog Search Challenge, LSC21. Taipei, Thailand.

[11] Emil Knudsen, Thomas Holstein Qvortrup, Omar Shahbaz Khan, and Björn Pór Jónsson. 2021. XOC at the Lifelog Search Challenge 2021: Interactive Learning on a Mobile Device. In Proceedings of the 2021 ACM Workshop on the Lifelog Search Challenge, LSC21. Taipei, Thailand.

[12] Andreas Leibetseder and Klaus Schoeffmann. 2021. lifeXplore at the Lifelog Search Challenge 2021. In Proceedings of the 2021 ACM Workshop on the Lifelog Search Challenge, LSC21. Taipei, Thailand.

[13] Jakub Lokoč, František Mejzlík, Patrik Veselý, Miroslav Kratochvíl, and Tomáš Souček. 2021. Enhanced SOMHunter for Known-item Search in Lifelog Data. In Proceedings of the 2021 ACM Workshop on the Lifelog Search Challenge, LSC21. Taipei, Thailand.

[14] Thao-Nhu Nguyen, Van-Tu Ninh, Tu-Khiem Le, Cathal Gurrin, Thanh-Binh Nguyen, Minh-Triet Tran, Annalina Caputo, and Graham Healy. 2021. LifeSeeker 3.0 : An Interactive Lifelog Search Engine for LSC'21. In Proceedings of the 2021 ACM Workshop on the Lifelog Search Challenge, LSC21. Taipei, Thailand.

[15] Luca Rossetto, Matthias Baumgartner, Ralph Gasser, Lucien Heitz, Ruijie Wang, and Abraham Bernstein. 2021. Exploring Graph-querying approaches in LifeGraph. In Proceedings of the 2021 ACM Workshop on the Lifelog Search Challenge, LSC21. Taipei, Thailand.

[16] Luca Rossetto, Ralph Gasser, Loris Sauter, Abraham Bernstein, and Heiko Schuldt. 2021. A system for interactive multimedia retrieval evaluations. In International Conference on Multimedia Modeling. Springer, 385-390.

[17] Jihye Shin, Alexandra Waldau, Aaron Duane, and Björn Pór Jónsson. 2021. PhotoCube at the Lifelog Search Challenge 2021. In Proceedings of the 2021 ACM Workshop on the Lifelog Search Challenge, LSC21. Taipei, Thailand.

[18] Florian Spiess, Ralph Gasser, Silvan Heller, Luca Rossetto, Loris Sauter, Milan van Zanten, and Heiko Schuldt. 2021. Exploring Intuitive Lifelog Retrieval and Interaction Modes in Virtual Reality with vitrivr-VR. In Proceedings of the 2021 ACM Workshop on the Lifelog Search Challenge, LSC21. Taipei, Thailand.

[19] Ly-Duyen Tran, Manh-Duy Nguyen, Nguyen Thanh Binh, Hyowon Lee, and Cathal Gurrin. 2020. Myscéal: An Experimental Interactive Lifelog Retrieval System for LSC'20. In Proceedings of the Third Annual Workshop on Lifelog Search Challenge (Dublin, Ireland) (LSC '20). Association for Computing Machinery, New York, NY, USA, 23-28.

[20] Ly-Duyen Tran, Manh-Duy Nguyen, Hyowon Lee, Thanh Binh Nguyen, and Cathal Gurrin. 2021. Myscéal 2.0: A Revised Experimental Interactive Lifelog Retrieval System for LSC'21. In Proceedings of the 2021 ACM Workshop on the Lifelog Search Challenge, LSC21. Taipei, Thailand.

[21] Hoang-Phuc Trang-Trung, Thanh-Cong Le, Mai-Khiem Tran, Van-Tu Ninh, TuKhiem Le, Cathal Gurrin, and Minh-Triet Tran. 2021. Flexible Interactive Retrieval SysTem 2.0 for Visual Lifelog Exploration at LSC 2021. In Proceedings of the 2021 ACM Workshop on the Lifelog Search Challenge, LSC21. Taipei, Thailand. 